\title{
Relevance of the three-dimensional Thirring coupling at finite temperature and density
}

\author{
R. Narayanan 1 \\ Department of Physics, Florida International University, Miami, Florida 33199, USA
}

(Received 4 June 2020; accepted 7 July 2020; published 20 July 2020)

\begin{abstract}
We studied the three-dimensional Thirring model in the limit of an infinite number of flavors at finite temperature and density. We calculated the number density as a function of temperature and the density at zero temperature serves as a relevant parameter. A three-dimensional free fermion gas behavior as the density at zero temperature approaches zero smoothly crosses over to a two-dimensional free fermion gas behavior as the density at zero temperature approaches infinity.
\end{abstract}

DOI: $10.1103 /$ PhysRevD.102.016014

\section{INTRODUCTION AND SUMMARY}

The three-dimensional Euclidean (two spatial and one thermal) Thirring model with $N$ flavors of two-component fermions would have been deemed nonrenormalizable by a standard power counting argument but it has been shown to be renormalizable in a $\frac{1}{N}$ expansion [1-5] implying that the Thirring coupling is relevant in the limit of large $N$. Recently, this strongly coupled theory at zero temperature and density has been extensively studied at finite values of $N$ to explore the possibility of mass generation [6-20]. With the possible exception of the $N=1$ model, a spontaneous generation of mass is most likely ruled out. A numerical analysis of QED in three dimensions has also resulted in similar observations [21-25] but monopoles present in QED can become relevant [26].

In this paper, we explore the relevance of the Thirring coupling in the large $N$ limit at finite temperature and density. We consider only the massless theory owing to the previous analysis of spontaneous mass generation. The physics at zero temperature has been briefly sketched out in [27]. The effective action in the large $N$ limit after introducing the standard vector auxiliary field is complex for a nonzero chemical potential and this leads us to perform a saddle point analysis. There are several saddle points at a fixed chemical potential and temperature but we will provide a graphical proof that only one particular saddle point dominates at all values of chemical potential and temperature.

Let $T$ and $\mu$ stand for the temperature and chemical potential measured in units of inverse of the Thirring

Published by the American Physical Society under the terms of the Creative Commons Attribution 4.0 International license. Further distribution of this work must maintain attribution to the author(s) and the published article's title, journal citation, and DOI. Funded by SCOAP ${ }^{3}$. coupling per flavor, $\lambda$. We will show that the number density at zero temperature in units of $\lambda$ is

$$
\mathbf{n}_{0}=\mu+2 \pi\left(1-\sqrt{1+\frac{\mu}{\pi}}\right),
$$

and can be used to set the chemical potential. The relevance of the Thirring coupling is already seen by noticing that the number density at zero temperature smoothly crosses over from $\frac{\mu^{2}}{4 \pi}$ as $\mu \rightarrow 0$ to $\mu$ as $\mu \rightarrow \infty$. Defining a reduced temperature by $T=\sqrt{4 \pi \mathbf{n}_{0}} t$ and writing the number density as a function of temperature as $\overline{\mathbf{n}}\left(\mathbf{n}_{0}, t\right) \mathbf{n}_{0}$, we will show that $\overline{\mathbf{n}}\left(\mathbf{n}_{0}, t\right)$ is the solution to

$$
\begin{aligned}
\overline{\mathbf{n}}\left(\mathbf{n}_{0}, t\right) & =8 t^{2} r_{2}\left(\frac{1+\sqrt{\frac{\mathbf{n}_{0}}{4 \pi}}\left[1-\overline{\mathbf{n}}\left(\mathbf{n}_{0}, t\right)\right]}{2 t}, 0\right), \\
1 & \leq \overline{\mathbf{n}}\left(\mathbf{n}_{0}, t\right) \leq 1+\sqrt{\frac{4 \pi}{\mathbf{n}_{0}}},
\end{aligned}
$$

where

$$
\begin{aligned}
r_{2}(u, \theta) & =\frac{\pi^{2}}{24}+\frac{u^{2}}{2}-\frac{\theta^{2}}{2}+\sum_{k=1}^{\infty}(-1)^{k} \frac{\left(e^{-2 k u}\right) \cos (2 k \theta)}{2 k^{2}} ; \\
u & >0 ; \quad-\frac{\pi}{2} \leq \theta<\frac{\pi}{2} .
\end{aligned}
$$

This result for the number density is plotted as a function of temperature in Fig. 1 and shows that it smoothly crosses over from a three-dimensional free fermion gas as $\mathbf{n}_{0} \rightarrow 0$ to a two-dimensional free fermion gas as $\mathbf{n}_{0} \rightarrow \infty$.

The rest of the paper is organized as follows. We set up our notation for the three-dimensional Thirring model and arrive at the saddle point equations in the limit of $N \rightarrow \infty$ in Sec. II. The saddle point that dominates at all chemical 


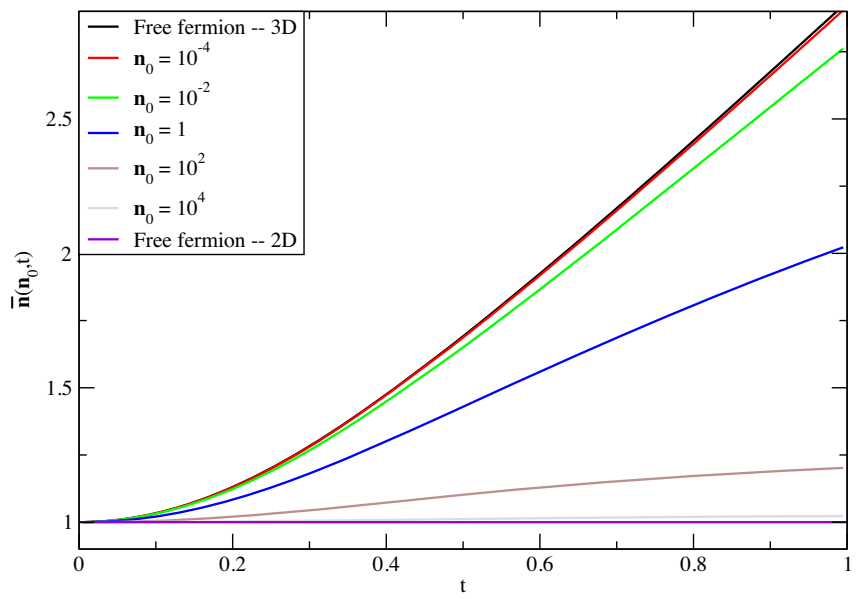

FIG. 1. Number density as a function of temperature for different choices of density at zero temperature.

potentials and temperatures is analyzed in Sec. III to obtain the main results stated above. Due to the involved interdependencies of the different saddle points at a fixed temperature and chemical potential, we revert to a graphical analysis in Sec. IV to show that the saddle point discussed in Sec. III dominates at all chemical potentials.

\section{THE THREE-DIMENSIONAL CONTINUUM THIRRING MODEL}

The action for the continuum Thirring model in three Euclidean dimensions is given by

$$
\begin{aligned}
S\left(\bar{\psi}_{i}, \psi_{i} ; \lambda\right)= & \int d^{3} x \sum_{i=1}^{N} \bar{\psi}_{i} D(\mu) \psi_{i} \\
& +\frac{1}{2 N \lambda} \int d^{3} x \sum_{k=1}^{3}\left(\sum_{i=1}^{N} \bar{\psi}_{i} \sigma_{k} \psi_{i}\right)^{2},
\end{aligned}
$$

where

$$
D(\mu)=\sum_{k=1}^{3} \sigma_{k} \partial_{k}+\sigma_{3} \mu
$$

is the Dirac operator acting on an irreducible twocomponent fermion in a $\ell^{2} \times \beta$ periodic box and $\mu$ is the chemical potential. The fermions obey periodic boundary conditions in the spatial directions and antiperiodic boundary conditions in the thermal direction. Upon introduction of a vector auxiliary field, $V_{k}(x)$, to replace the four-Fermi interaction and a subsequent integration of the fermions results in

$S\left(V_{k}, \lambda\right)=N\left[\frac{\lambda}{2}\left(\int d^{3} x \sum_{k} V_{k}^{2}\right)-\ln \operatorname{det}\left(D\left(V_{k}\right)\right)\right]$,

where

$$
D\left(V_{k}\right)=\sum_{k=1}^{2} \sigma_{k}\left(\partial_{k}+i V_{k}\right)+\sigma_{3} \mu
$$

With $V_{k} \rightarrow-V_{k}$ and $x_{k} \rightarrow-x_{k}$, we see that $\mu \rightarrow-\mu$. Therefore, it is sufficient to consider a positive value for the chemical potential in our analysis.

Assuming translational invariance to hold in the large $N$ limit, ${ }^{1}$ we will analyze the action per flavor with the auxiliary field restricted to constants,

$V_{1}(x)=\frac{2 \pi h_{1}}{\ell} ; \quad V_{2}(x)=\frac{2 \pi h_{2}}{\ell} ; \quad V_{3}(x)=\frac{2 \pi h_{3}}{\beta}$.

The minimum will occur at $h_{1}=h_{2}=0$ in the $\ell \rightarrow \infty$ limit. One can use standard formulas in [34] to perform the sum over momentum in the $\beta$ direction and the resulting action density (per unit spatial volume) per flavor is

$$
\begin{aligned}
S\left(h_{3} ; T, \mu\right)= & 2 \pi^{2} T h_{3}^{2}-\frac{2 T^{2}}{\pi} \int_{0}^{\infty} d x x \ln \\
& \times \frac{4 \cosh \left(x+i \pi h_{3}+\frac{\mu}{2 T}\right) \cosh \left(x-i \pi h_{3}-\frac{\mu}{2 T}\right)}{e^{2 x}} .
\end{aligned}
$$

The integration variable is related to the spatial momentum by $x=\frac{\beta p}{2}=\frac{p}{2 T}$ and we have set $T$ and $\mu$ in units of $\lambda$.

The action density is complex and we will perform a saddle point analysis. We elevate $\left(2 \pi h_{3}\right)$ to a complex variable $z=\gamma+i \frac{\delta}{T}$ and define

$$
\begin{array}{ll}
\omega=u+i \theta ; \quad u=\frac{\mu-\delta}{2 T} ; \quad & \theta \in\left[\frac{-\pi}{2}, \frac{\pi}{2}\right] ; \\
z=-2 i\left(\omega-\frac{\mu}{2 T}+i n \pi\right) ; & n \in \mathbf{I} .
\end{array}
$$

The action density can be written in terms of polylogarithms [35] as

$$
\begin{gathered}
S(n, \omega ; T, \mu)=S^{b}(n, \omega ; T, \mu)+S^{f}(\omega ; T, \mu) ; \\
S^{b}(n, \omega ; T, \mu)=-2 T\left(\omega-\frac{\mu}{2 T}+i n \pi\right)^{2} ; \\
S^{f}(\omega ; T, \mu)=\frac{T^{2}}{2 \pi}\left[\operatorname{Li}_{3}\left(-e^{2 \omega}\right)+\mathrm{Li}_{3}\left(-e^{-2 \omega}\right)\right] .
\end{gathered}
$$

The partition function is given by

$$
Z(T, \mu)=\sum_{n=-\infty}^{\infty} \int_{-\frac{i \pi}{2}}^{\frac{i \pi}{2}} d \omega e^{-N \ell^{2} S(n, \omega ; T, \mu)}
$$

\footnotetext{
${ }^{1}$ This assumption is reasonable if one compares it to the Gross-Neveu model where translational invariance holds in three dimensions [28] even though it does not hold in two dimensions [29-33].
} 
The saddle points are given by $\frac{d S(n, \omega ; T, \mu)}{d \omega}=0$ which we will label by $\omega_{n}$ or $\left(\theta_{n}, \delta_{n}\right)$ with the possibility that more than one saddle point exists at a fixed $n$.

The number density per flavor is given by

$$
\begin{aligned}
\mathbf{n}(T, \mu) & =\frac{T}{\ell^{2} N} \frac{d \ln Z(T, \mu)}{d \mu}=-\frac{1}{2 Z(T, \mu)} \sum_{n=-\infty}^{\infty} \int_{-\frac{i \pi}{2}}^{\frac{i \pi}{2}} d \omega \frac{d S_{f}(n, \omega ; T, \mu)}{d \omega} e^{-N \ell^{2} S(n, \omega ; T, \mu)} \\
& =-\frac{1}{2 Z(T, \mu)} \sum_{n=-\infty}^{\infty} \int_{-\frac{i \pi}{2}}^{\frac{i \pi}{2}} d \omega\left(4 T\left(\omega-\frac{\mu}{2 T}+i n \pi\right)+\frac{d S(n, \omega ; T, \mu)}{d \omega}\right) e^{-N \ell^{2} S(z ; T, \mu)} .
\end{aligned}
$$

\section{A. Equations for saddle points}

The complex valued equation for the saddle point using polylogarithm identities is

$$
\begin{aligned}
\frac{\mu}{2 T} & =\omega_{n}+i n \pi+\frac{T}{4 \pi}\left[\operatorname{Li}_{2}\left(-e^{-2 \omega_{n}}\right)-\operatorname{Li}_{2}\left(-e^{2 \omega_{n}}\right)\right] \\
& = \begin{cases}\frac{\pi T}{24}+\omega_{n}+i n \pi+\frac{T}{2 \pi} \omega_{n}^{2}+\frac{T}{2 \pi} \sum_{k=1}^{\infty}(-1)^{k} \frac{e^{-2 k \omega_{n}}}{k^{2}} ; & u_{n}>0 \\
-\frac{\pi T}{24}+\omega_{n}+i n \pi-\frac{T}{2 \pi} \omega_{n}^{2}-\frac{T}{2 \pi} \sum_{k=1}^{\infty}(-1)^{k} \frac{e^{2 k \omega_{n}}}{k^{2}} ; & u_{n}<0 .\end{cases}
\end{aligned}
$$

For the purpose of analysis, we separate the above equation into its real and imaginary part as

$$
\begin{aligned}
\frac{\pi\left(\theta_{n}+n \pi\right)}{T} & =r_{1}\left(\left|u_{n}\right|, \theta_{n}\right)=-\left|u_{n}\right| \theta_{n}+\sum_{k=1}^{\infty}(-1)^{k} \frac{e^{-2 k\left|u_{n}\right|} \sin \left(2 k \theta_{n}\right)}{2 k^{2}} ; \\
\frac{\pi \mu}{2 T^{2}} & =\frac{u_{n}}{\left|u_{n}\right|}\left[\frac{\pi\left|u_{n}\right|}{T}+r_{2}\left(\left|u_{n}\right|, \theta_{n}\right)\right] \\
r_{2}\left(\left|u_{n}\right|, \theta_{n}\right) & =\frac{\pi^{2}}{24}+\frac{u_{n}^{2}}{2}-\frac{\theta_{n}^{2}}{2}+\sum_{k=1}^{\infty}(-1)^{k} \frac{\left(e^{-2 k\left|u_{n}\right|}\right) \cos \left(2 k \theta_{n}\right)}{2 k^{2}} .
\end{aligned}
$$

The real and imaginary parts of the action density needed to study the dominance of one saddle point over another are

$$
\begin{aligned}
S_{R}^{b}(n, \theta, \delta ; T, \mu) & =2 T(\theta+n \pi)^{2}-\frac{\delta^{2}}{2 T} \\
S_{R}^{f}(\theta, \delta ; T, \mu) & =\frac{2 T^{2}}{\pi}\left[|u|\left(\theta^{2}-\frac{\pi^{2}}{12}\right)-\frac{|u|^{3}}{3}+\sum_{k=1}^{\infty}(-1)^{k} \frac{e^{-2 k|u|} \cos (2 k \theta)}{2 k^{3}}\right] \\
S_{I}^{b}(n, \theta, \delta ; T, \mu) & =2 \delta(\theta+n \pi) \\
S_{I}^{f}(\theta, \delta ; T, \mu) & =-\frac{u}{|u|} \frac{2 T^{2}}{\pi}\left[\frac{\theta^{3}}{3}-\frac{\pi^{2} \theta}{12}+u^{2} \theta+\sum_{k=1}^{\infty}(-1)^{k} \frac{e^{-2 k|u|} \sin (2 k \theta)}{2 k^{3}}\right] .
\end{aligned}
$$

Referring to Eq. (A3), we note that the derivative of $r_{1}(|u|, \theta)$ with respect to $\theta$ at $\theta=0$ is negative. The derivative can become zero at most once for $|\theta|<\frac{\pi}{2}$, if $|u|<u_{0}=\frac{1}{2} \ln \frac{3+\sqrt{5}}{2}$. Referring to Eq. (A1), we conclude that $r_{1}(|u|, \theta)$ is positive for $-\frac{\pi}{2} \leq \theta<0$ and negative for $0<\theta \leq \frac{\pi}{2}$. There is always a saddle point with $n=0$ and it has $\theta_{0}=0$. For $n \neq 0$, the saddle point, if one exists, will be in the region $-\frac{\pi}{2} \leq \theta_{n}<0$ if $n>0$ and will be in the region $0<\theta_{n} \leq \frac{\pi}{2}$ if $n<0$.
Assuming the saddle point at $n=0$ dominates, the result for the number density will be

$$
\mathbf{n}(T, \mu)=\delta_{0} .
$$

Let us assume we have a solution to the saddle point equations given by $\left(\theta_{n}, \delta_{n}\right)$ with $n>0$. Since

$$
\begin{aligned}
& r_{1}\left(\left|u_{n}\right|,-\theta_{n}\right)=-r_{1}\left(\left|u_{n}\right|, \theta_{n}\right) ; \\
& r_{2}\left(\left|u_{n}\right|,-\theta_{n}\right)=r_{2}\left(\left|u_{n}\right|, \theta_{n}\right), \quad u_{-n}=u_{n},
\end{aligned}
$$


we can conclude that $\left(\theta_{-n}, \delta_{-n}\right)=\left(-\theta_{n}, \delta_{n}\right)$ is a saddle point with $-n<0$. It follows from Eq. (17) that

$$
\begin{aligned}
S_{R}\left(n, \theta_{n}, \delta_{n} ; T, \mu\right) & =S_{R}\left(-n, \theta_{-n}, \delta_{-n} ; T, \mu\right) ; \\
S_{I}\left(n, \theta_{n}, \delta_{n} ; T, \mu\right) & =-S_{I}\left(-n, \theta_{-n}, \delta_{-n} ; T, \mu\right) .
\end{aligned}
$$

If this pair of saddle points were to dominate over the solitary one at $n=0$, the number density will be

$$
\mathbf{n}(T, \mu)=\delta_{n}-2\left(\theta_{n}+n \pi\right) T \tan \left[N \ell^{2} S_{I}\left(n, \theta_{n}, \delta_{n} ; T, \mu\right)\right]
$$

which does not have a smooth $N \rightarrow \infty$ limit.

\section{ANALYSIS ASSUMING THE SADDLE POINT AT $n=0$ DOMINATES}

The only equation is

$$
\frac{\pi \mu}{2 T^{2}}=\frac{u_{0}}{\left|u_{0}\right|}\left[\frac{\pi\left|u_{0}\right|}{T}+r_{2}\left(\left|u_{0}\right|, 0\right)\right] .
$$

Referring to Eq. (A2), we have $r_{2}\left(\left|u_{0}\right|, 0\right)>0$ which results in

$$
u_{0}>0 ; \quad \delta_{0}=\frac{2 T^{2}}{\pi} r_{2}\left(\frac{\mu-\delta_{0}}{2 T}, 0\right) .
$$

Rewriting the saddle point equation as

$$
\frac{\mu}{T^{2}}-\frac{2 u_{0}}{T}=\frac{2}{\pi} r_{2}\left(u_{0}, 0\right) ; \quad u_{0}=\frac{\mu-\delta_{0}}{2 T}>0,
$$

we see there is one and only one solution for $u_{0}$ such that $0 \leq u_{0} \leq \frac{\mu}{2 T}\left(0 \leq \delta_{0} \leq \mu\right)$. Using Eq. (A3),we obtain

$$
\frac{\partial \delta_{0}}{\partial T}=\frac{2 T}{\pi} \frac{2 r_{2}\left(u_{0}, 0\right)+u_{0} d_{1}\left(u_{0}, 0\right)}{1-\frac{T}{\pi} d_{1}\left(u_{0}, 0\right)} .
$$

We note from Eqs. (A2) and (A3) that

$$
\begin{aligned}
& d_{1}\left(u_{0}, 0\right)<0 \\
& 2 r_{2}\left(u_{0}, 0\right)+u_{0} d_{1}\left(u_{0}, 0\right) \\
& \quad=u_{0} \ln \left(1+e^{-2 u_{0}}\right)+4 \int_{0}^{u_{0}} d x \frac{x}{1+e^{2 x}}>0 .
\end{aligned}
$$

Therefore we conclude that $\frac{\partial \delta_{0}}{\partial T}>0$ and $\delta_{0}$ is a monotonically increasing function of temperature at a fixed chemical potential.

Assuming that the saddle point at $n=0$ dominates at all chemical potentials and temperatures, the number density is given by $\mathbf{n}=\delta_{0}$. Since

$$
\lim _{T \rightarrow 0} \frac{2 T^{2}}{\pi} r_{2}\left(u_{0}, 0\right)=\frac{\left(\mu-\delta_{0}\right)^{2}}{4 \pi},
$$

the saddle point equation as $T \rightarrow 0$ is

$$
\mathbf{n}_{0}=\frac{\left(\mu-\mathbf{n}_{0}\right)^{2}}{4 \pi}
$$

which results in the number density at $T=0$ to be

$$
\mathbf{n}_{0}=\mu+2 \pi\left(1-\sqrt{1+\frac{\mu}{\pi}}\right) .
$$

We can trade the chemical potential for $\mathbf{n}_{0}$ using

$$
\mu=\mathbf{n}_{0}+\sqrt{4 \pi \mathbf{n}_{0}} .
$$

To see the relevance of the Thirring coupling, we define a reduced temperature by $T=\sqrt{4 \pi \mathbf{n}_{0}} t$ and write the number density at any temperature as $\left[\overline{\mathbf{n}}\left(\mathbf{n}_{0}, t\right) \mathbf{n}_{0}\right]$, noting that $\overline{\mathbf{n}}$ will depend on $\mathbf{n}_{0}$ in addition to $t$. It is this dependence that shows the relevance of the Thirring coupling. Referring to Eqs. (23) and (30) we arrive at

$$
\overline{\mathbf{n}}\left(\mathbf{n}_{0}, t\right)=8 t^{2} r_{2}\left(\frac{1+\sqrt{\frac{\mathbf{n}_{0}}{4 \pi}}\left[1-\overline{\mathbf{n}}\left(\mathbf{n}_{0}, t\right)\right]}{2 t}, 0\right) .
$$

One can either use Eq. (16) or Eq. (A2) and see that the above equation reduces to a free fermion behavior in three dimensions as $\mathbf{n}_{0} \rightarrow 0$. Using Eqs. (A2) and (24), the asymptotic behavior in $t$ at a fixed $\mathbf{n}_{0}$ is

$$
\overline{\mathbf{n}}\left(\mathbf{n}_{0}, t\right)=1+\sqrt{\frac{4 \pi}{\mathbf{n}_{0}}}-\frac{\pi\left(1+\sqrt{\frac{4 \pi}{\mathbf{n}_{0}}}\right)}{(\ln 2) \sqrt{4 \pi \mathbf{n}_{0}}} \frac{1}{t}+O\left(t^{-2}\right) .
$$

Since the number density monotonically increases with temperature at a fixed $\mathbf{n}_{0}$, it is bounded by

$$
1 \leq \overline{\mathbf{n}}\left(\mathbf{n}_{0}, t\right) \leq 1+\sqrt{\frac{4 \pi}{\left|\mathbf{n}_{0}\right|}} .
$$

The density does not change with temperature in the limit $\mathbf{n}_{0} \rightarrow \infty$ and this is the free fermion behavior in two dimensions. The subleading term for small $t$ is

$$
\overline{\mathbf{n}}\left(\mathbf{n}_{0}, t\right)=1+\frac{\pi^{2}}{3} \frac{1}{1+\sqrt{\frac{\mathbf{n}_{0}}{\pi}}} t^{2}+O\left(t^{3}\right) .
$$

A plot of $\overline{\mathbf{n}}\left(\mathbf{n}_{0}, t\right)$ as a function of $t$ has already been shown in Fig. 1 at several different values of $\mathbf{n}_{0}$ to demonstrate the crossover from three-dimensional free 
fermion behavior as $\mathbf{n}_{0} \rightarrow 0$ to two-dimensional free fermion behavior as $\mathbf{n}_{0} \rightarrow \infty$.

\section{NONDOMINANCE OF THE SADDLE POINTS AT $n \neq 0$}

We will only consider $n>0$ since we have shown at the end of Sec. II A that saddle points at $n$ and $(-n)$ are paired. Then every solution has to satisfy $-\frac{\pi}{2} \leq \theta_{n} \leq 0$. We will fix the temperature and use $\theta_{n}$ instead of chemical potential since it is more convenient from the viewpoint of solving the saddle point equations. We will graphically demonstrate the nondominance of the saddle points at $n \neq 0$ by defining

$$
\begin{aligned}
& \Delta\left(n, \theta_{n}, T\right) \\
& =\frac{S_{R}\left(n, \theta_{n}, \delta_{n}\left(\theta_{n}\right) ; \mu\left(\theta_{n}\right), T\right)-S_{R}\left(0,0, \delta_{0}\left(\theta_{n}\right) ; \mu\left(\theta_{n}\right), T\right)}{2 T n^{2} \pi^{2}}
\end{aligned}
$$

and showing that it remains positive at all $n, T$ and allowed values of $\theta_{n} \in\left[-\frac{\pi}{2}, 0\right]$. To this end we will use the following steps:

(1) We will show that $\mu \rightarrow \infty$ as $\theta_{n} \rightarrow 0$ at all temperatures.

(2) There exists a temperature $T_{0}(n)$ above which a certain region given by $-\frac{\pi}{2}<\theta_{l}(T)<\theta_{n}<\theta_{r}(T)<0$ has no solution to the saddle point equations. The chemical potential will monotonically increase from 0 to $\infty$ as $\theta_{n}$ increases from $\theta_{r}(T)$ to 0 and it will monotonically increase from 0 to a finite nonzero value as $\theta_{n}$ decreases from $\theta_{l}(T)$ to $-\frac{\pi}{2}$. Furthermore, $\theta_{l}\left(T_{0}(n)\right)=\theta_{r}\left(T_{0}(n)\right)=\theta_{0}(n)$.

(3) There exists a temperature $T_{1}(n)$ below which the chemical potential will monotonically decrease from $\infty$ to a finite nonzero value as $\theta_{n}$ decreases from 0 to $-\frac{\pi}{2}$. In other words there will be a region of chemical potential given by $0 \leq \mu<\mu_{1}(T)$ for which there is no solution to the saddle point equations. Furthermore, $\mu_{1}\left(T_{1}(n)\right)=0$.

(4) We will explicitly study the case of zero temperature and the case of zero chemical potential.

(5) Even though $\Delta\left(n, \theta_{n}, T\right)$ will not be monotonic in $\theta_{n}$ at a fixed $n$ and temperature, it reaches an absolute minimum at $\theta=-\frac{\pi}{2}$ at all $n$ and temperatures. We will study $\Delta\left(n,-\frac{\pi}{2}, T\right)$ as a function of $T$ and show that the minimum at each $n$ is larger than zero for all $n$.

$$
\text { A. } \theta_{n} \rightarrow 0_{-} \Rightarrow \mu \rightarrow \infty
$$

Keeping terms to relevant orders in $\theta_{n}$ and using Eq. (A3), the first equation in Eq. (16) results in

$$
\left|u_{n}\right|=-\frac{n \pi^{2}}{T \theta_{n}}-\frac{\pi}{T}+O\left(e^{\frac{1}{\theta_{n}}}\right) .
$$

Inserting this into the second equation in Eq. (16) results in

$$
u_{n}>0 ; \quad \mu=\frac{n^{2} \pi^{3}}{\theta_{n}^{2}}+\frac{\pi T^{2}}{12}-\pi+O\left(\theta_{n}^{2}\right) .
$$

If we use the diverging chemical potential with the leading correction into Eq. (24), we find that $u_{0}=u_{n}$ up to the two correction terms. Therefore, we arrive at

$$
\Delta\left(n, 0_{-}, T\right)=1
$$

and the saddle point at $n=0$ dominates as $\mu \rightarrow \infty$ for all values of temperature.

\section{B. $T_{0}(n)$}

Noting that $d_{2}(|u|, \theta)>0$ for $-\frac{\pi}{2}<\theta<0$, the righthand side of the first equation in Eq. (16) monotonically increases with $|u|$ at a fixed $\theta$. Therefore, at every value of $\theta$, the lowest value of the right-hand side is given by $r_{1}(0, \theta)$. If the temperature is above a certain value, we will have a region of $\theta$ where there is no solution to the first equation in Eq. (16). This transition temperature is the solution to

$$
\begin{aligned}
\frac{\pi}{T_{0}(n)}\left(\theta_{0}(n)+n \pi\right) & =r_{1}\left(0, \theta_{0}(n)\right) ; \\
\frac{\pi}{T_{0}(n)} & =-\ln \left[2 \cos \theta_{0}(n)\right],
\end{aligned}
$$

which results in

$$
\begin{array}{lcc}
\theta_{0}(1)=-0.372690809 \pi ; & \theta_{0}(2)=-0.350144201 \pi ; & \theta_{0}(5)=-0.339555189 \pi \\
T_{0}(1)=12.563174152 ; & T_{0}(2)=2 \times 16.123729198 ; & T_{0}(5)=5 \times 18.139848147 ;
\end{array}
$$

and

$$
\theta_{0}(\infty)=\frac{\pi}{3} ; \quad \lim _{n \rightarrow \infty} \frac{T_{0}(n)}{n}=\frac{\pi^{2}}{r_{1}\left(0, \frac{\pi}{3}\right)}=19.448615248 .
$$

Since $u_{n}=0$ at this temperature, $T_{0}(n)$, and $\theta_{0}(n)$ we have $r_{2}\left(0, \theta_{0}(n)\right)=0$ and $\mu=0$ at this point. 


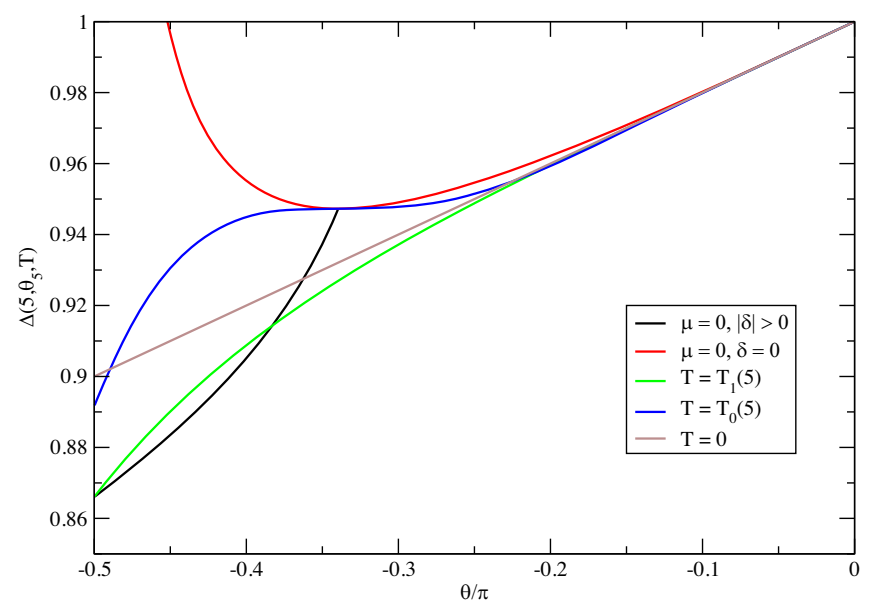

FIG. 2. The action at the $n=5$ saddle point is taken as a typical example since it has all the features for a generic $n$ and it is compared with the $n=0$ saddle point to show that the $n=0$ saddle point dominates at all temperatures and chemical potential. This plot shows the comparison at some specific values of temperature and also at zero chemical potential.

After fixing the temperature, we choose a $\theta_{n}$ in the allowed region and solve for $\left|u_{n}\right|$ using the first equation in Eq. (16) and then solve for the chemical potential using the second equation. Referring to Eq. (A3), we note that $d_{2}(|u|, \theta)$ remains non-negative in $-\frac{\pi}{2} \leq \theta_{n} \leq 0$ and we conclude that $r_{2}(|u|, \theta)$ is a monotonically increasing function in $-\frac{\pi}{2} \leq \theta_{n} \leq 0$. Since we are only considering $\mu>0$, we can conclude from the second equation in Eq. (16) that $u_{n}>0$ if $\left[\frac{\pi\left|u_{n}\right|}{T}+r_{2}\left(\left|u_{n}\right|, \theta_{n}\right)\right]>0$ and $u_{n}<0$ if $\left[\frac{\pi\left|u_{n}\right|}{T}+r_{2}\left(\left|u_{n}\right|, \theta_{n}\right)\right]<0$. Furthermore, we can have a solution to the saddle point equations with $n>0$ and $\mu=0$ only if $\left[\frac{\pi\left|u_{n}\right|}{T}+r_{2}\left(\left|u_{n}\right|, \theta\right)\right]=0$. We have used $n=5$ as a typical value of $n$ and plotted $\Delta\left(5 ; \theta_{5}, T_{0}(5)\right)$ as a blue curve in Fig. 2. We see it remains positive in the entire range of $\theta_{5}$ showing that the saddle at $n=0$ dominates over $n=5$ at $T_{0}(5)$.

\section{C. $T_{1}(n)$}

Consider moving down from $\theta_{n}=0$. Noting that $u_{n}$ is positive to start with and noting that $d_{2}(|u|, \theta)$ is positive in $-\frac{\pi}{2} \leq \theta_{n} \leq 0$, we conclude from Eq. (A5) that $\mu$ decreases as $\theta_{n}$ moves down from zero as long as $u_{n}$ remains positive. At $\theta_{n}=-\frac{\pi}{2}$, the first equation in Eq. (16) reduces to

$$
\left|u_{n}\right|=\frac{(2 n-1) \pi}{T}
$$

and the condition for $\left[\frac{\pi\left|u_{n}\right|}{T}+r_{2}\left(\left|u_{n}\right|,-\frac{\pi}{2}\right)\right]$ to remain positive is

$$
\frac{\pi^{2}}{T^{2}}\left(4 n^{2}-1\right)-\frac{\pi^{2}}{6}+\sum_{k=1}^{\infty} \frac{e^{-\frac{2(2 n-1) k \pi}{T}}}{k^{2}}>0 .
$$

This gives the condition for $\mu=0$ to occur for $T>T_{n}(n)$ with

$$
\begin{array}{ll}
T_{1}(1)=4.654727000 ; & T_{1}(2)=2 \times 4.987847524 ; \\
T_{1}(5)=5 \times 5.044788571 ; & \lim _{n \rightarrow \infty} \frac{T_{1}(n)}{n}=5.028967463 .
\end{array}
$$

We have plotted $\Delta\left(5 ; \theta_{5}, T_{1}(5)\right)$ as a red curve in Fig. 2 . We see it remains positive in the entire range of $\theta_{5}$ showing that the saddle at $n=0$ dominates over $n=5$ at $T_{1}(5)$.

\section{D. $T=0$}

Let us consider the limit $T \rightarrow 0$. The two saddle point equations in Eq. (16) sequentially result in

$$
\begin{aligned}
\lim _{T \rightarrow 0} T\left|u_{n}\right| & =-\frac{\pi\left(\theta_{n}+n \pi\right)}{\theta_{n}} ; \\
\lim _{T \rightarrow 0} T^{2}\left[\frac{\pi\left|u_{n}\right|}{T}+r_{2}\left(\left|u_{n}\right|, \theta_{n}\right)\right] & =\frac{\pi^{2}\left(n^{2} \pi^{2}-\theta_{n}^{2}\right)}{2 \theta_{n}^{2}}>0 ;
\end{aligned}
$$

and we obtain

$$
\mu=\frac{\pi\left(n^{2} \pi^{2}-\theta_{n}^{2}\right)}{\theta_{n}^{2}} ; \quad \delta_{n}=\frac{\pi\left(\theta_{n}+n \pi\right)^{2}}{\theta_{n}^{2}} .
$$

With this choice of $\mu$, the saddle point solution with $n=0$ is given by

$\lim _{T \rightarrow 0} T u_{0}=-\frac{\pi\left(\theta_{n}+n \pi\right)}{\theta_{n}} ; \quad \delta_{0}=\frac{\pi\left(\theta_{n}+n \pi\right)^{2}}{\theta_{n}^{2}}$,

and we conclude

$$
\Delta\left(n, \theta_{n}, 0\right)=1+\frac{\theta_{n}}{n \pi} .
$$

We have plotted $\Delta\left(5, \theta_{5}, 0\right)$ for reference in Fig. 2 as a brown line.

$$
\text { E. } \boldsymbol{\mu}=\mathbf{0}
$$

If $T>T_{1}(n)$ we have a saddle point solution with $\mu=0$ and $n>0$. Setting $\mu=0$, we first note by referring to Eq. (23) that the saddle point with $n=0$ corresponds to $u_{0}=0$ and therefore $\delta_{0}=0$ and the real part of the action at the $n=0$ saddle point is [referring to Eq. (17)]

$$
S_{R}(0,0,0 ; 0, T)=\frac{2 T^{2}}{\pi} \sum_{k=1}^{\infty} \frac{(-1)^{k}}{2 k^{3}}=-\frac{3 \zeta(3)}{4 \pi} T^{2} .
$$

Referring to Eq. (16), the solution to the saddle points at $n>0$ is given by 
$f\left(\left|u_{n}\right|, \theta_{n}\right)=\left|u_{n}\right| r_{1}\left(\left|u_{n}\right|, \theta_{n}\right)+\left(\theta_{n}+n \pi\right) r_{2}\left(\left|u_{n}\right|, \theta_{n}\right)=0$.

Viewing the above equation as a function of $\left|u_{n}\right|$ at a fixed $\theta_{n}$ we note that $\left|u_{n}\right|=0$ is a solution to the above equation. In this case, $\delta_{n}=0$ and the temperature as a function of $\theta_{n}$ from the first equation in Eq. (16) is

$$
T_{n}\left(\theta_{n}\right)=\frac{\pi\left(\theta_{n}+n \pi\right)}{r_{1}\left(0, \theta_{n}\right)}
$$

and

$$
\begin{aligned}
\Delta\left(n, \theta_{n}, T_{n}\left(\theta_{n}\right)\right) & =\left[\frac{\theta_{n}}{n \pi}+1\right]^{2}+\frac{T_{n}\left(\theta_{n}\right)}{2 n^{2} \pi^{3}} J_{f}\left(\theta_{n}\right) ; \\
J_{f}(\theta) & =\sum_{k=1}^{\infty}(-1)^{k} \frac{\cos (2 k \theta)-1}{k^{3}} .
\end{aligned}
$$

Noting that

$$
J_{f}\left(-\frac{\pi}{2}\right)=\frac{7 \zeta(3)}{4}>0 ; \quad J_{f}(0)=0
$$

and noting that

$$
\frac{d J_{f}(\theta)}{d \theta}=2 \mathrm{Cl}_{2}(\pi-2 \theta) \leq 0
$$

where $\mathrm{Cl}_{2}$ is the Clausen function of order 2 , we conclude that $J_{f}(\theta)$ is non-negative in $-\frac{\pi}{2}<\theta_{n}<0$. Therefore, these saddle points at $n>0$ and $\mu=0$ do not dominate over $n=0$. We have plotted $\Delta\left(5, \theta_{5}, T\right)$ for $\mu=0$ and $\delta_{5}=0$ as a red curve in Fig. 2. Note that the divergence in $\Delta\left(5, \theta_{5}, T\right)$ as $\theta_{5} \rightarrow-\frac{\pi}{2}$ can be seen from Eqs. (52) and (53).

There is another solution to Eq. (50) with $\left|u_{n}\right|>0$ if $T_{1}(n)<T<T_{0}(n)$ and $\theta_{n}<\theta_{0}(n)$. To see this note that the derivative of Eq. (50) with respect to $\left|u_{n}\right|$ gives us

$$
\begin{aligned}
\frac{\partial f\left(\left|u_{n}\right|, \theta_{n}\right)}{\partial\left|u_{n}\right|}= & r_{1}\left(\left|u_{n}\right|, \theta_{n}\right)+\left|u_{n}\right| d_{2}\left(\left|u_{n}\right|, \theta_{n}\right) \\
& -\left(\theta_{n}+n \pi\right) d_{1}\left(\left|u_{n}\right|, \theta_{n}\right) .
\end{aligned}
$$

The first two terms are positive in the range $-\frac{\pi}{2}<\theta_{n}<0$. Furthermore, $d_{1}\left(\left|u_{n}\right|, \theta_{n}\right)$ is positive only if $-\frac{\pi}{2}<\theta_{n}<-\frac{\pi}{6}$. We can see that $f\left(0, \theta_{n}\right)=0$ and $f\left(\left|u_{n}\right|, \theta_{n}\right)$ goes as $\left[\frac{n \pi-\theta_{n}}{2} u_{n}^{2}\right]$ as $\left|u_{n}\right| \rightarrow \infty$. If $\frac{\partial f\left(0, \theta_{n}\right)}{\partial\left|u_{n}\right|}>0$ the only solution to Eq. (50) is $\left|u_{n}\right|=0$. The condition for $\frac{\partial f\left(0, \theta_{n}\right)}{\partial\left|u_{n}\right|}=0$ is the same as Eq. (39) and therefore we conclude that $-\frac{\pi}{2}<$ $\theta_{n}<\theta_{0}(n)$ for a solution to Eq. (50) to exist with $\left|u_{n}\right| \neq 0$. We have plotted $\Delta\left(5, \theta_{5}, T\right)$ for $\mu=0$ and $\delta_{5} \neq 0$ as a black curve in Fig. 2 and it meets the red curve at $\theta_{0}(5)$. Note that the temperature along the black curve changes from $T=$ $T_{1}(5)$ at $\theta_{5}=-\frac{\pi}{2}$ to $T=T_{0}(5)$ at $\theta_{5}=\theta_{0}(5)$. A generic
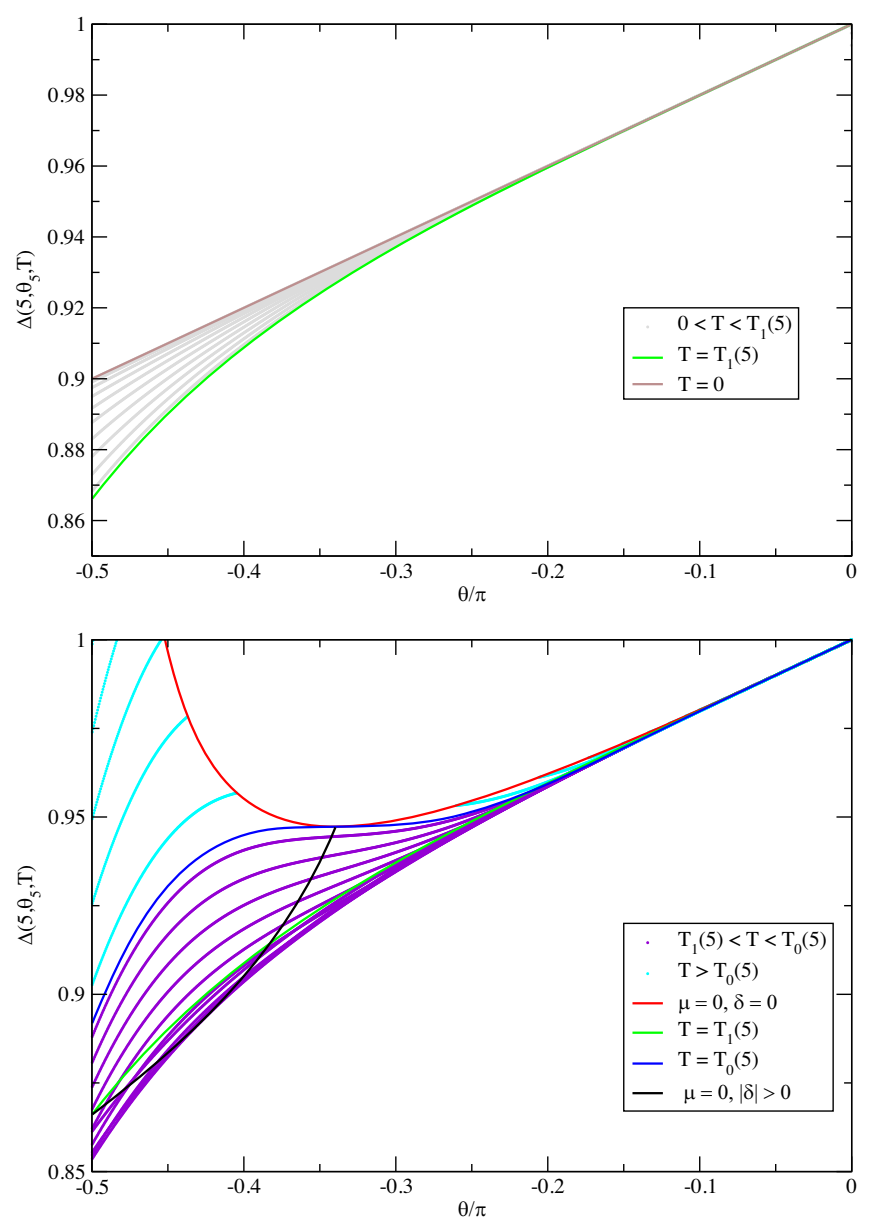

FIG. 3. The top plot shows that the difference between $T=0$ and $T=T_{1}(5)$ is bounded by the two extremes. The bottom plot shows that the difference between $T=T_{1}(5)$ and $T=T_{0}(5)$ moves smoothly from one end to the other but there is a temperature in between the two ends where the difference is smallest as a function of $\theta_{n}$. On the other hand, the difference for $T>T_{0}(5)$ is bounded from below by $T=T_{0}(5)$.

feature of the curves shown in Fig. 2 is the intersection of the black curve $\left(\mu=0\right.$ and $\left.\delta_{5} \neq 0\right)$ with the green curve $\left(T_{1}(5)\right)$ at a value of $\theta_{n}$ away from $-\frac{\pi}{2}$. The temperature and chemical potential on the two curves at the intersection point are different but yield the same value for $\Delta\left(n, \theta_{n}, T\right)$.

\section{F. Analysis at $\boldsymbol{\theta}_{\boldsymbol{n}}=-\frac{\pi}{2}$}

The two plots in Fig. 3 show the behavior of $\Delta\left(5, \theta_{5}, T\right)$ as a function of $\theta_{5}$ over the entire range of $T$. For $T<T_{1}(n)$, we find a solution in the entire range of $\theta_{n}$ with $\mu$ monotonically increasing from a positive finite value at $\theta_{n}=-\frac{\pi}{2}$ to a divergence at $\theta_{n}=0$. The gray points in the top plot of Fig. 3 show $\Delta\left(1, \theta_{1}, T\right)$ for $T<T_{1}(n)$ and all points lie between the line at $T=0$ and $T=T_{1}(1)$ and moves continuously from one to the other as $T$ changes. For $T_{1}(n)<T<T_{0}(n)$, we again find a solution in the 


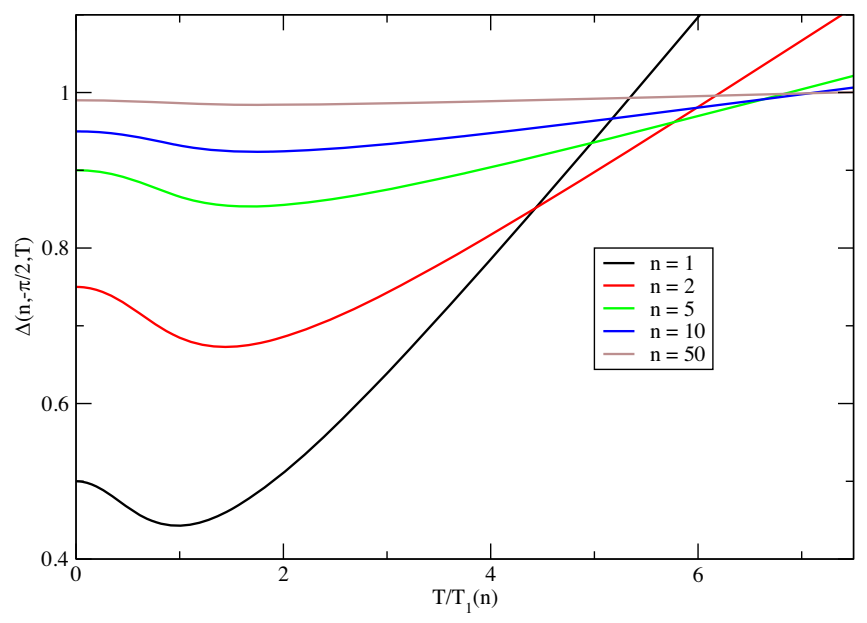

FIG. 4. The difference in the action between $n>0$ and $n=0$ at $\theta_{n}=-\frac{\pi}{2}$ is plotted as a function of $T / T_{1}(n)$.

entire range of $\theta_{n}$. But we find a $\theta_{0}(T)$ at which $\mu=0$. The chemical potential monotonically decreases from a positive finite value at $\theta_{n}=-\frac{\pi}{2}$ to zero at $\theta_{n}=\theta_{0}(T)$ and then monotonically increases to a divergence at $\theta_{n}=0$. Furthermore, $\theta_{0}\left(T_{1}(n)\right)=-\frac{\pi}{2}$ and $\theta_{0}\left(T_{0}(n)\right)=\theta_{0}(n)$. These features are shown in the bottom plot of Fig. 3 . The curves for $\Delta\left(5, \theta_{5}, T\right)$ at a fixed $T$ in $T_{1}(5)<T<$ $T_{0}(5)$ are shown by violet points. Note that there is a range of $T$ starting from $T_{1}(5)$ where the curve intersects the black curve $\left(\mu=0\right.$ and $\left.\delta_{5} \neq 0\right)$ at two points. Only one of the intersection points has the same temperature and chemical potential. Initially, it is the intersection point closer to $\theta_{5}=-\frac{\pi}{2}$ until the violet curve is tangential to the black curve. After that, the intersection point closer to $\theta_{0}(5)$ is where the temperature and chemical potential matches. For $T>T_{0}(5)$, there will be a solution only if $\theta_{5}$ does not belong to the interval $\left(\theta_{l}(T), \theta_{r}(T)\right)$ which can be obtained by setting the left-hand side to $T$ in Eq. (51). The chemical potential will monotonically decrease from a positive finite value at $\theta_{n}=-\frac{\pi}{2}$ to zero at $\theta_{l}(T)$ and then monotonically increase from zero at $\theta_{r}(T)$ to a divergence at $\theta_{n}=0$. These features are also shown in the bottom plot of Fig. 3 and we see that $\Delta\left(5, \theta_{5}, T\right)$ for $T>T_{0}(5)$ shown by cyan points lies above the curve at $T=T_{0}(5)$ and has two parts. The boundary of the two parts is the red curve with $\mu=0$ and $\delta=0$.

The graphical analysis discussed before specifically for $n=5$ enables us to conclude that $\Delta\left(5, \theta_{5}, T\right)$ reaches a minimum at $\theta_{5}=-\frac{\pi}{2}$ at any fixed temperature. We therefore plot $\Delta\left(n,-\frac{\pi}{2}, T\right)$ as a function of $T$ for $n=1,2,5,10$, 50 in Fig. 4. The minimum occurs very close to $T=T_{1}(1)$ but moves to a higher temperature with respect to $T_{1}(n)$ as $n$ is increased. The global minimum occurs at a finite value of $T / T_{1}(n)$ even as $n \rightarrow \infty$. This plot along with the analysis done specifically for $n=5$ in Figs. 2 and 3 serves to graphically prove our main point- the saddle point at $n=0$ dominates at all chemical potentials and temperatures.

\section{CONCLUSIONS}

We have studied the three-dimensional (two spatial and one thermal) Euclidean Thirring model at finite temperature and chemical model in the limit of infinite number of flavors and our aim was to understand the relevance of the Thirring coupling. The effective action is complex and we had to perform an extensive graphical analysis to show that one particular saddle point dominates over all other possible saddle points at all chemical potential and temperature. The relevance of the Thirring coupling is seen in the dependence of the behavior of the number density as a function of temperature on different values of the number density at zero temperature. The number density as a function of temperature behaves like a three-dimensional free fermion gas as the number density at zero temperature approaches zero and it behaves like a two-dimensional free fermion gas as the number density at zero temperature approaches infinity. The number density at a fixed nonzero value at zero temperature is a monotonically increasing function of temperature reaching a finite value at infinite temperature. The function for any given nonzero value of density at zero temperature smoothly interpolates between the two extremal behaviors at zero number density and infinite number density at zero temperature.

\section{ACKNOWLEDGMENTS}

The author acknowledges partial support by the NSF under Grant No. PHY-1913010.

\section{APPENDIX: SOME USEFUL PROPERTIES OF $r_{1}(u, \theta)$ AND $r_{2}(u, \theta)$}

We note that

$$
r_{1}(|u|, 0)=0 ; \quad r_{1}\left(|u|, \pm \frac{\pi}{2}\right)=\mp \frac{\pi|u|}{2} .
$$

Using Riemann and Dirchlet zeta function formulas [35], we also note that

$$
\begin{array}{r}
r_{2}(|u|, 0)=\frac{u^{2}}{2}+\int_{0}^{|u|} d x \ln \left(1+e^{-2 x}\right) ; \\
r_{2}\left(|u|, \pm \frac{\pi}{2}\right)=\frac{u^{2}}{2}+\int_{0}^{|u|} d x \ln \left(1-e^{-2 x}\right) .
\end{array}
$$

The derivatives of $r_{1}(|u|, \theta)$ and $r_{2}(|u|, \theta)$ with respect to $|u|$ and $\theta$ are 


$$
\begin{aligned}
\frac{\partial r_{1}(|u|, \theta)}{\partial \theta} & =-\frac{\partial r_{2}(|u|, \theta)}{\partial|u|} \\
& =-\frac{1}{2} \ln [2(\cosh (2 u)+\cos (2 \theta))]=d_{1}(u, \theta), \\
\frac{\partial r_{1}(|u|, \theta)}{\partial|u|} & =\frac{\partial r_{2}(|u|, \theta)}{\partial \theta}=-\tan ^{-1}(\tanh |u| \tan \theta) \\
& =d_{2}(|u|, \theta) .
\end{aligned}
$$

Referring to the solutions as $\theta_{n}(\mu), \delta_{n}(\mu)$ and $u_{n}(\mu)$ we compute expressions for the derivative of $\delta_{n}$ with respect to $\mu$. Noting that

$$
\frac{\partial\left|u_{n}\right|}{\partial \mu}=\frac{u_{n}}{\left|u_{n}\right|} \frac{1}{2 T}\left(1-\frac{\partial \delta_{n}}{\partial \mu}\right)
$$

and referring to Eq. (16), we obtain

$$
\begin{aligned}
\frac{\partial \theta_{n}}{\partial \mu} & =\frac{u_{n}}{\left|u_{n}\right|} \frac{\pi d_{2}}{2 T^{2}\left[\left(\frac{\pi}{T}-d_{1}\left(\left|u_{n}\right|, \theta_{n}\right)\right)^{2}+d_{2}^{2}\left(\left|u_{n}\right|, \theta_{n}\right)\right]} ; \\
\frac{\partial \delta_{n}}{\partial \mu} & =1-\frac{\pi}{\pi-T d_{1}\left(\left|u_{n}\right|, \theta_{n}\right)+T \frac{d_{2}^{2}\left(\left|u_{n}\right| \theta_{n}\right)}{\left.\frac{\pi}{T}-d_{1}\left|u_{n}\right| \theta_{n}\right)}} .
\end{aligned}
$$

[1] G. Parisi, Nucl. Phys. B100, 368 (1975).

[2] S. Hikami and T. Muta, Prog. Theor. Phys. 57, 785 (1977).

[3] Z. Yang, University of Texas, Report No. UTTG-40-90, 1990.

[4] M. Gomes, R. Mendes, R. Ribeiro, and A. da Silva, Phys. Rev. D 43, 3516 (1991).

[5] S. Hands, Phys. Rev. D 51, 5816 (1995).

[6] L. Del Debbio and S. Hands, Phys. Lett. B 373, 171 (1996).

[7] S. Hands (UKQCD Collaboration), in International Workshop on Perspectives of Strong Coupling Gauge Theories (SCGT 96) (1996), pp. 383-389.

[8] L. Del Debbio, S. Hands, and J. Mehegan (UKQCD Collaboration), Nucl. Phys. B502, 269 (1997).

[9] L. Del Debbio and S. Hands, Nucl. Phys. B552, 339 (1999).

[10] S. Hands and B. Lucini, Phys. Lett. B 461, 263 (1999).

[11] S. Christofi, S. Hands, and C. Strouthos, arXiv:hep-lat/ 0703016.

[12] S. Christofi, S. Hands, and C. Strouthos, Phys. Rev. D 75, 101701 (2007).

[13] H. Gies and L. Janssen, Phys. Rev. D 82, 085018 (2010).

[14] L. Janssen and H. Gies, Phys. Rev. D 86, 105007 (2012).

[15] D. Schmidt, B. Wellegehausen, and A. Wipf, Proc. Sci., LATTICE2015 (2016) 050 [arXiv:1511.00522].

[16] B. H. Wellegehausen, D. Schmidt, and A. Wipf, Phys. Rev. D 96, 094504 (2017).

[17] S. Hands, EPJ Web Conf. 175, 11008 (2018).

[18] S. Hands, Phys. Rev. D 99, 034504 (2019).

[19] S. Hands, Proc. Sci., Confinement2018 (2018) 221.

[20] J. J. Lenz, B. H. Wellegehausen, and A. Wipf, Phys. Rev. D 100, 054501 (2019).
[21] N. Karthik and R. Narayanan, Phys. Rev. D 93, 045020 (2016).

[22] N. Karthik and R. Narayanan, Phys. Rev. D 94, 065026 (2016).

[23] N. Karthik and R. Narayanan, Phys. Rev. D 96, 054509 (2017).

[24] N. Karthik and R. Narayanan, Proc. Sci., LATTICE2016 (2016) 245 [arXiv:1610.09355].

[25] N. Karthik and R. Narayanan, Phys. Rev. Lett. 121, 041602 (2018).

[26] N. Karthik and R. Narayanan, arXiv:1908.05500.

[27] M. Goykhman, J. High Energy Phys. 07 (2016) 034.

[28] R. Narayanan, Phys. Rev. D 101, 096001 (2020).

[29] U. Wolff, Phys. Lett. 157B, 303 (1985).

[30] M. Thies and K. Urlichs, Phys. Rev. D 67, 125015 (2003).

[31] P. de Forcrand and U. Wenger, Proc. Sci., LAT2006 (2006) 152 [arXiv:hep-lat/0610117].

[32] L. Pannullo, J. Lenz, M. Wagner, B. Wellegehausen, and A. Wipf, Proc. Sci., LATTICE2019, 063 (2019) [arXiv:1909 .11513].

[33] J. Lenz, L. Pannullo, M. Wagner, B. Wellegehausen, and A. Wipf, Phys. Rev. D 101, 094512 (2020).

[34] I. Gradshteyn and I. Ryzhik, Table of Integrals, Series, and Products (Academic Press, New York, 2007).

[35] DLMF, NIST Digital Library of Mathematical Functions, edited by F. W. J. Olver, A. B. Olde Daalhuis, D. W. Lozier, B. I. Schneider, R. F. Boisvert, C. W. Clark, B. R. Miller, B. V. Saunders, H. S. Cohl, and M. A. McClain, http://dlmf .nist.gov/. 